Arq. Bras. Med. Vet. Zootec., v.65, n.4, p.955-960, 2013

\title{
Índice gonadossomático e correlações entre dimensões e peso testiculares na codorna japonesa (Coturnix coturnix japonica) aos 60 dias de idade
}

\author{
[Gonadosomatic index and correlations between testicular dimensions and weight in \\ Japanese quail (Coturnix coturnix japonica) at 60 days old] \\ L.L. Lanna ${ }^{1}$, F.A. Soares ${ }^{1}$, T.M. Santos ${ }^{1}$, J.N. Oliveira ${ }^{1}$, A.P. Marques Júnior ${ }^{2}$ \\ ${ }^{1}$ Instituto Federal do Norte de Minas Gerais - IFNMG - Salinas, MG \\ ${ }^{2}$ Escola de Veterinária da Universidade Federal de Minas Gerais - UFMG - Belo Horizonte, MG
}

\begin{abstract}
RESUMO
As dimensões e o peso testicular foram avaliados em 25 machos adultos de codornas japonesas (Coturnix coturnix japonica) e correlacionados entre si, a fim de se buscar obter informações que possibilitem a criação de um parâmetro preditivo da capacidade de produção espermática. As aves foram abatidas e os testículos removidos para mensuração e pesagem. As dimensões e o peso entre os testículos esquerdo e direito foram comparados. Os parâmetros testiculares foram correlacionados entre si por meio da determinação do coeficiente de correlação linear. O índice gonadossomático também foi determinado, indicando alocação de $3,68 \%$ do peso corporal em testículos nos machos avaliados, resultado maior do que os descritos na literatura. Apesar da diferença de formato entre os testículos esquerdo e direito, o peso foi semelhante, sugerindo que não há diferença na capacidade de produção espermática entre eles. Diferença estatística entre os valores biométricos indica que o testículo esquerdo é mais curto e mais arredondado, enquanto o direito é mais longo e estreito. $\mathrm{O}$ peso corporal apresentou baixa correlação com o peso testicular, 0,14 e 0,12 para os testículos esquerdo e direito, respectivamente. Os parâmetros que melhor se correlacionaram com o peso testicular foram a largura e a espessura. Considerando-se a identificação de um parâmetro único para comparação entre machos, a espessura do testículo esquerdo apresentou coeficiente de correlação linear de 0,89 com o somatório do peso dos dois testículos. Com base nessa informação, métodos não invasivos, como a ultrassonografia, poderiam ser utilizados para estimar o potencial reprodutivo e auxiliar na comparação de machos de codornas japonesas em núcleos de seleção de aves elite, contribuindo para melhoramento genético da espécie.
\end{abstract}

Palavras-chave: dimensões testiculares, peso testicular, índice gonadossomático, codorna japonesa

\begin{abstract}
The size and testis weight were evaluated and correlated in 25 adult male Japanese quail (Coturnix coturnix japonica), seeking information to enable the development of a predictive parameter of sperm production capacity. The birds were slaughtered and testes removed for measuring and weighing. The size and testis weight were compared between the left and right. The testicular parameters were correlated by determining the linear correlation coefficient. The gonadosomatic index was also determined, indicating the allocation of $3.68 \%$ of body weight in the testes, greater than described in literature. Despite the difference in shape between the left and right testis, weight was similar, suggesting that there is no difference in the capacity of sperm production amongst them. A statistical difference between the biometric values indicates that the left testicle is shorter and more rounded, while the right is longer and narrower. Body weight showed a low correlation with the testicular weight, 0.14 and 0.12 for the left and right testes, respectively. The parameters that best correlated with testicular weight were the width and thickness. Given the identification of a single parameter for comparison of males, the thickness of the left testis showed linear correlation coefficient of 0.89 with the sum of the weight of both testicles. From this information, noninvasive methods such as ultrasound could be used to estimate the reproductive potential and ease the comparison of male Japanese quail in elite selection nuclei, contributing to the genetic improvement of the species.
\end{abstract}

Keywords: testicular dimensions, testicular weight, gonadosomatic index, Japanese quail

Recebido em 31 de outubro de 2012

Aceito em 8 de abril de 2013

E-mail: leonardollanna@gmail.com 


\section{INTRODUÇÃO}

A coturnicultura, um ramo da avicultura industrial, tem apresentado crescimento acentuado nas últimas décadas e conquistado adeptos, tendo a codorna japonesa (Coturnix coturnix japonica) como a mais difundida entre os criadores no Brasil e no mundo. Entre os principais motivos que tornam a criação comercial de codornas tão atrativa, destacam-se: o baixo investimento para implantação; a capacidade de abrigar grande número de aves em pequeno espaço físico; a alta produtividade; a simplicidade de mão de obra; e o rápido retorno do capital investido (Albino e Barreto, 2003).

Apesar dos recentes avanços e do potencial de crescimento da coturnicultura, aspectos básicos da morfofisiologia do aparelho genital da codorna japonesa, alguns com possíveis implicações práticas, ainda não estão completamente descritos. Atualmente, nenhum tipo de parâmetro é utilizado como preditivo da fertilidade em machos de aves (Kirby et al., 2001; Obidi et al., 2008), como o que ocorre, por exemplo, em bovinos, em que se utiliza o perímetro escrotal (Hafez e Hafez, 2003). Isso dificulta o processo de seleção reprodutiva, tão importante para garantir altas taxas de fertilidade em plantéis de aves domésticas (Amann, 1999). A localização intra-abdominal dos testículos é a explicação para a inexistência de tal parâmetro, porém os adventos da ultrassonografia podem contribuir para a utilização prática de tais indicadores (Bath e Chaudhari, 2002).

Índice gonadossomático é o valor que expressa a porcentagem do peso corporal alocada nas gônadas, sendo representado pelo somatório do peso dos testículos, dividido pelo peso corporal e multiplicado por 100 (Kenagy e Trombulak, 1986). Clulow e Jones (1982) relataram que os testículos de codorna japonesa representam 2,26\% do peso corporal, sendo proporcionalmente grandes quando comparados aos da maioria dos mamíferos. Isso favorece a alta frequência de cópulas resultante do hábito poligâmico e a competição espermática sugerida por alguns autores (Schein et al., 1972; Birkhead et al., 1993).

A produção espermática por grama de testículo é relativamente constante para uma espécie ou linhagem. Portanto, machos com maior massa testicular apresentam maior capacidade de produção espermática. Estudos demonstraram que o peso do testículo está diretamente relacionado com a produção espermática diária (França e Russell, 1998) e a reserva espermática (Bath e Chaudhari, 2002). A maioria dos autores considera que o testículo esquerdo de aves é maior que o direito, sendo responsável por maior produção de células espermáticas (Amoroso et al., 2008; Obidi et al., 2008). Bath e Chaudhari (2002) relataram que o peso corporal está positivamente relacionado com a produção espermática em galos.

Alguns pesquisadores tentaram promover a seleção de aves com maior capacidade de produção espermática baseando-se em características sexuais secundárias e comportamento sexual, o que indicaria a concentração de testosterona circulante. Porém, logo se percebeu que algumas aves mantinham produção hormonal mesmo em condições de espermatogênese deficiente, contraindicando a utilização de tal referencial. A identificação de um parâmetro biométrico testicular que se correlacione com a capacidade de produção espermática pode contribuir para a seleção reprodutiva em aves (Kirby et al., 1998).

Tendo em vista o exposto e o potencial de expansão da coturnicultura no Brasil, objetivouse com o presente trabalho correlacionar as dimensões testiculares e o peso corporal com o peso testicular, além de determinar o índice gonadossomático na codorna japonesa aos 60 dias de idade.

\section{MATERIAL E MÉTODOS}

Foram utilizados 25 machos de codorna japonesa, com 60 dias de idade, amostrados de um lote de incubação no Setor de Coturnicultura do Instituto Federal Norte de Minas Gerais (IFNMG) - Campus Salinas, com eclosão dos ovos na segunda quinzena de abril. As aves foram criadas em box medindo $2,10 \times 2,70 \mathrm{~m}$, com piso de concreto forrado com cama de maravalha. Durante os primeiros 14 dias de vida, as aves foram submetidas à iluminação diária de 24 horas, tendo à disposição bebedouros do tipo copo de pressão e comedouros tipo bandeja. A partir do $15^{\circ}$ dia, o ambiente passou a receber apenas iluminação natural (12 horas de luz por 
dia), com bebedouros pendulares e comedouros tubulares.

Após 35 dias de idade e sexagem, os machos foram transferidos para gaiolas com dimensões de $25 \times 33 \times 18 \mathrm{~cm}$, equipadas com bebedouro tipo nipple e calha de alimentação, na proporção de um macho para três fêmeas. Durante esse período, foi realizado aumento gradativo de iluminação artificial, até um total de 17 horas de luz por dia ao redor dos 40 dias de idade. Durante todas as etapas, foram oferecidas água e dieta comercial balanceada ad libitum. O projeto foi aprovado pelo Comitê de Ética em Experimentação Animal (Cetea) da Universidade Federal de Minas Gerais (UFMG), protocolo 060/11.

Aos 60 dias de idade, os machos foram abatidos pelo método de deslocamento cervical. As aves foram pesadas, posicionadas em decúbito dorsal para retirada de penas da região ventral e incisão de pele e musculatura para acesso à cavidade celomática. Após remoção das vísceras, os testículos foram individualizados e removidos para avaliação macroscópica, mensuração com paquímetro digital e pesagem em balança analítica digital com precisão de três casas decimais. As dimensões mensuradas foram comprimento, largura (da margem epididimária para a lateral), espessura (da margem ventral para a dorsal) e perímetro. Para essa última, utilizou-se barbante, posicionado ao redor da maior seção transversal do testículo, cortado e esticado para aferição do perímetro.

O índice gonadossomático (IGS) foi calculado pela somatória do peso dos testículos (Ptest), dividido pelo peso corporal (Pcorp) e multiplicado por 100 , conforme a fórmula:

$$
I G S=(\text { Ptest } / \text { Pcorp }) \times 100 .
$$

Os dados são apresentados na forma de média e desvio-padrão. Foram estabelecidos coeficiente correlação linear de Pearson (r) entre as dimensões testiculares, peso corporal e peso testicular. A significância das correlações e as dimensões entre os testículos direito e esquerdo foram comparadas com o teste $\mathrm{t}$ de Student, ao nível de significância de $1 \%$.

\section{RESULTADOS E DISCUSSÃO}

O testículo esquerdo e o direito apresentaram peso semelhante, diferentemente de autores que consideram o testículo esquerdo maior na codorna japonesa (Amoroso et al., 2008). Quanto ao formato, foi observado que o testículo esquerdo apresenta largura, espessura e perímetro maiores, sendo mais arredondado que o direito, que é mais alongado, conforme evidenciado pela diferença significativa entre comprimento, largura, espessura e perímetro entre eles (Tab. 1). A Fig. 1 ilustra a diferença de formato entre os dois testículos. Apesar da diferença na morfologia, os resultados indicam que a capacidade de produção espermática é semelhante entre os testículos, uma vez que ela está diretamente relacionada com o peso testicular (França e Russell, 1998), o que difere do encontrado em galos por Obidi et al. (2008), os quais verificaram maior reserva espermática na gônada esquerda.

Tabela 1. Peso, comprimento, largura, espessura e perímetro dos testículos esquerdo e direito na codorna japonesa (Coturnix coturnix japonica) aos 60 dias de idade (média \pm desvio-padrão)

\begin{tabular}{cccccc} 
& Peso $(\mathrm{g})$ & $\begin{array}{c}\text { Comprimento } \\
(\mathrm{mm})\end{array}$ & Largura $(\mathrm{mm})$ & $\begin{array}{c}\text { Espessura } \\
(\mathrm{mm})\end{array}$ & $\begin{array}{c}\text { Perímetro } \\
(\mathrm{mm})\end{array}$ \\
\hline $\begin{array}{l}\text { Testículo } \\
\text { esquerdo }\end{array}$ & $2,48 \pm 0,70$ & $21,73 \underline{ \pm 1,86 \mathrm{a}}$ & $15,38 \pm 1,55 \mathrm{a}$ & $14,15 \pm 1,54 \mathrm{a}$ & $46,72 \pm 4,42 \mathrm{a}$ \\
$\begin{array}{c}\text { Testículo } \\
\text { direito }\end{array}$ & $2,47 \pm 0,65$ & $24,29 \pm 1,88 \mathrm{~b}$ & $14,00 \pm 1,75 \mathrm{~b}$ & $12,92 \pm 1,43 \mathrm{~b}$ & $43,13 \pm 4,36 \mathrm{~b}$ \\
\hline
\end{tabular}

Letras distintas em uma mesma coluna indicam diferença estatística no teste $\mathrm{t}$ de Student $(\mathrm{P}<0,01)$.

Para o testículo esquerdo, todas as dimensões mensuradas foram altamente correlacionadas com o peso testicular, com coeficientes de correlação linear acima de 0,80 (Tab. 2), o que indica a possibilidade de utilização de qualquer uma dessas medidas para predição da capacidade de produção espermática do testículo esquerdo de codornas japonesas. 


\section{Lanna et al.}

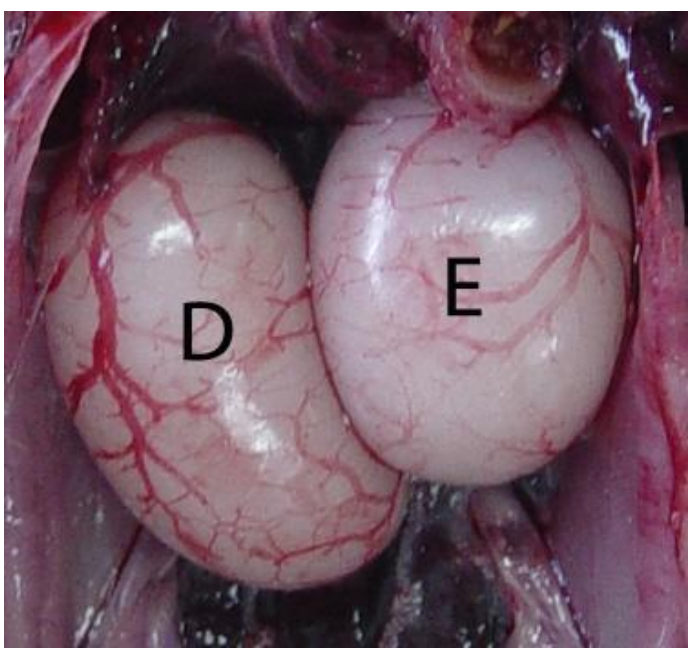

Figura 1. Formato dos testículos esquerdo e direito em codorna japonesa (Coturnix coturnix japonica) aos 60 dias de idade. O testículo esquerdo (E) é arredondado e curto, enquanto o direito (D) é alongado e estreito.

Considerando-se o testículo direito, o comprimento se mostrou parâmetro menos adequado para estimativa do peso testicular, com menor coeficiente de correlação linear, enquanto a largura, a espessura e o perímetro apresentaram maior correlação (Tab. 2). Levando em consideração que o perímetro do testículo direito é o parâmetro que melhor se correlaciona ao peso testicular e que a perímetro apresenta alta correlação com a largura e a espessura (Tab. 2), especula-se a praticidade de se mensurar um destes dois últimos parâmetros para comparação entre aves utilizadas como reprodutores.

$\mathrm{O}$ índice gonadossomático encontrado no presente estudo foi de $3,68 \%$ para codornas japonesas aos 60 dias de idade. Esse resultado é maior do que o encontrado em outras pesquisas, tanto na codorna japonesa, 2,26\% (Clulow e Jones, 1982), quanto na variedade italiana, 2,8\%, sendo esta última no Brasil (Orsi et al., 2005). Especula-se a influência de fatores genéticos, climáticos ou ambientais nessa diferença, porém os dados e o delineamento utilizados impossibilitam qualquer afirmação. $O$ tamanho proporcionalmente grande dos testículos dá suporte à teoria de que fotoperíodo artificial em regiões tropicais possibilita a exploração reprodutiva de codornas, mesmo no outono, época em que o experimento foi conduzido (Orsi et al., 2005).

Tabela 2. Coeficiente de correlação linear (r) entre parâmetros dos testículos esquerdo e direito na codorna japonesa (Coturnix coturnix japonica) aos 60 dias de idade

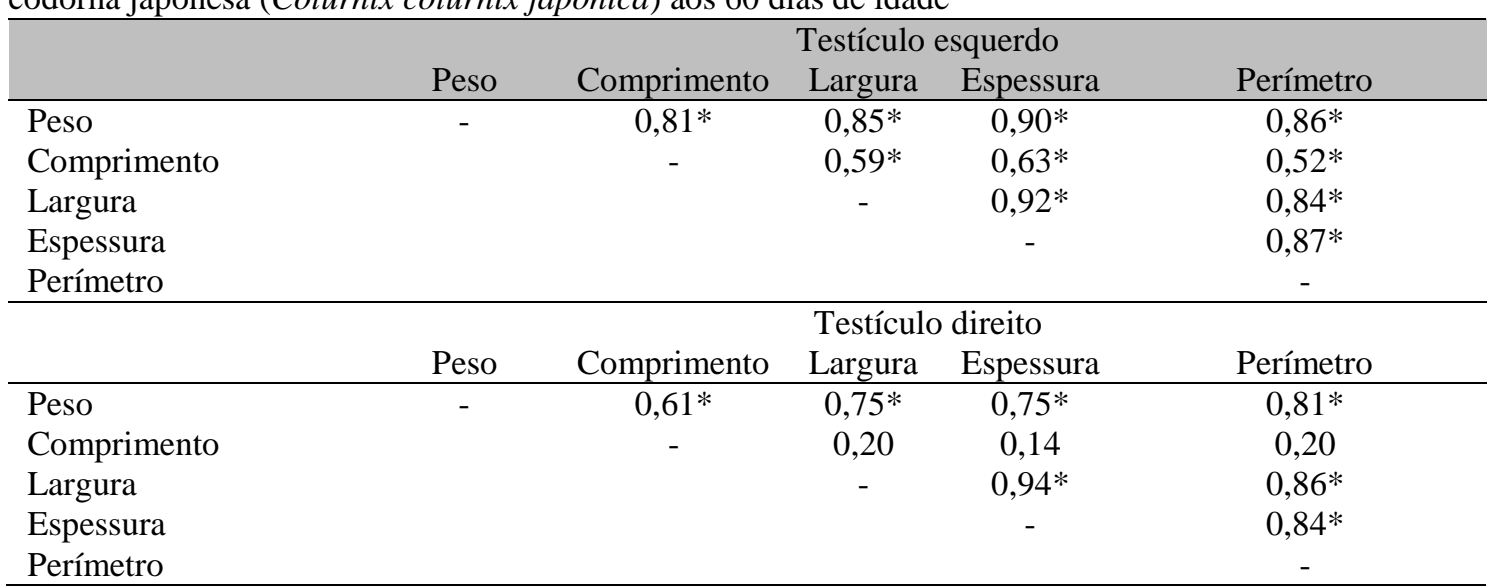

*Correlação de Pearson significativa ao teste $\mathrm{t}(\mathrm{P}<0,01)$.

O peso corporal apresentou baixa correlação com o peso testicular, com coeficiente linear de 0,14 para o testículo esquerdo e 0,12 para o direito, contrariando o observado em galos (Bath e Chaudhari, 2002) e reforçando a necessidade de utilização de parâmetros mais acurados na avaliação reprodutiva, como mensuração das dimensões testiculares. $\mathrm{Na}$ Tab. 3 constam as correlações entre os valores biométricos e o peso para cada um dos testículos. 
Tabela 3. Coeficiente de correlação linear (r) entre peso testicular e peso corporal, comprimento, largura, espessura e perímetro testiculares para os testículos esquerdo e direito na codorna japonesa (Coturnix coturnix japonica) aos 60 dias de idade

\begin{tabular}{|c|c|c|c|c|c|}
\hline & $\begin{array}{l}\text { Peso } \\
\text { corporal }\end{array}$ & $\begin{array}{l}\text { Comprimento } \\
\text { testicular }\end{array}$ & $\begin{array}{l}\text { Largura } \\
\text { testicular }\end{array}$ & $\begin{array}{l}\text { Espessura } \\
\text { testicular }\end{array}$ & $\begin{array}{l}\text { Perímetro } \\
\text { testicular }\end{array}$ \\
\hline $\begin{array}{l}\text { Peso do testículo } \\
\text { esquerdo }\end{array}$ & 0,14 & $0,81^{*}$ & $0,85^{*}$ & $0,90^{*}$ & $0,86^{*}$ \\
\hline $\begin{array}{l}\text { Peso do testículo } \\
\text { direito }\end{array}$ & 0,12 & $0,61^{*}$ & $0,75^{*}$ & $0,75^{*}$ & $0,81^{*}$ \\
\hline
\end{tabular}

*Correlação de Pearson significativa ao teste $\mathrm{t}(\mathrm{P}<0,01)$.

Em razão do manejo trabalhoso de seleção de reprodutores de codorna japonesa em núcleos de melhoramento genético com lotes maiores, avaliou-se também a correlação linear entre os parâmetros dos testículos esquerdo e direito com a massa testicular total, visando definir um único parâmetro a ser mensurado com métodos não invasivos, como a ultrassonografia (Tab. 4).

Tabela 4. Coeficiente de correlação linear (r) entre a massa testicular total e os parâmetros biométricos na codorna japonesa (Coturnix coturnix japonica) aos 60 dias de idade

\begin{tabular}{lcccc} 
& $\begin{array}{c}\text { Comprimento } \\
\text { testicular }\end{array}$ & Largura testicular & $\begin{array}{c}\text { Espessura } \\
\text { testicular }\end{array}$ & $\begin{array}{c}\text { Perímetro } \\
\text { testicular }\end{array}$ \\
\hline Testículo esquerdo & $0,74^{*}$ & $0,85^{*}$ & $0,89 *$ & $0,88^{*}$ \\
Testículo direito & $0,62^{*}$ & $0,68^{*}$ & $0,61^{*}$ & $0,73^{*}$ \\
\hline
\end{tabular}

*Correlação de Pearson significativa ao teste $\mathrm{t}(\mathrm{P}<0,01)$.

Tendo em vista o elevado coeficiente de correlação linear e a relativa simplicidade em se obter tal medida, a largura e a espessura do testículo esquerdo foram consideradas os melhores parâmetros a serem avaliados, caso fosse necessário optar por um único para mensurações com recursos como a ultrassonografia. $\mathrm{O}$ coeficiente de determinação $\left(\mathrm{r}^{2}\right)$ para ambas as características, próximo de 0,80 , indica que $80 \%$ da variação da massa testicular total na codorna japonesa aos 60 dias de idade são explicados por essa variável. Dessa maneira, especula-se a possibilidade de seleção reprodutiva por meio da mensuração não invasiva do eixo curto do testículo esquerdo, ou seja, largura ou espessura.

\section{CONCLUSÕES}

Apesar da diferença de formato, o peso dos testículos esquerdo e direito é semelhante, o que sugere contribuição equitativa para a produção espermática na codorna japonesa. A alta correlação entre a largura e a espessura e o peso testicular indica a possibilidade de se comparar o potencial reprodutivo de machos por meio da mensuração delas. $\mathrm{O}$ índice gonadossomático encontrado é maior do que a média encontrada por outros autores.

\section{AGRADECIMENTOS}

Ao apoio financeiro do Pibic e Pibic-Jr/Fapemig/CNPq.

\section{REFERÊNCIAS}

ALBINO, L.F.T.; BARRETO, S.L.T. Criação de codornas para produção de ovos e carne. Viçosa: Editora Aprenda Fácil, 2003. 268p.

AMANN, R.P. Lessons for the poultry industry gleaned from experiences with other commodity species. Poultry Sci., v.78, p.419-427, 1999.

AMOROSO, L.; ARTONI, S.M.B.; MORAES, V.M.B. et al. Influência da espermatogênese e dos níveis de testosterona no aspecto reprodutivo de codornas. Rev. Bras. Zootec., v.37, p.61-66, 2008.

BATH, G.S.; CHAUDHARI, S.U.R. Sperm reserves and its relationship to parameters of the testis, epididymis and vas deferens of local cocks in the Sahel region of Nigeria. Intern. J. Agric. Biol., v.4, p.561-564, 2002. 
BIRKHEAD, T.R.; BRISKIE, J.V.; MOLLER, A.P. Male sperm reserves and copulation frequency in birds. Behav. Ecol. Sociobiol., v.32, p.85-93, 1993.

CLULOW, J.; JONES, R.C. Production, transport, maturation, storage and survival of spermatozoa in the male Japanese quail, Coturnix coturnix. J. Reprod. Fert., v.64, p.259266, 1982.

FRANÇA, L.R.; RUSSELL, L.D. The testis of domestic animals. In: REGADERA, J.; MARTINEZ-GARCIA, F. (Ed.). Male reproduction: a multidisciplinary overview. Madrid: Churchill Livingstone, 1998. p.197-219.

HAFEZ, E.S.E.; HAFEZ, B. Reprodução Animal. 7.ed. South Carolina: Kiawah Island, 2003. 513p.

KENAGY, G.J.; TROMBULAK, S.C. Size and function of mammalian testes in relation to body size. J. Mamm., v.67, p.1-22, 1986.
KIRBY, J.D.; RORIE, R.W.; MELNYCHUK, V.L. et al. Ultrasound as a tool to assess reproductive status in poultry. Poultry Sci. Suppl., v.80, p.177, 2001.

KIRBY, J.D.; RORIE, R.W.; WASHINGTON, J. et al. Use of ultrasound to assess testis development in broiler breeder males. Poultry Sci. Suppl., v.77, p.91, 1998.

OBIDI, J.A.; ONYEANUSI, B.I.; AYO, J.O. et al. Determination of gonadal sperm/spermatid reserves in Shikabrown breeder cocks. Intern. J. Poultry Sci., v.7, p.1200-1203, 2008.

ORSI, A.M.; STEFANINI, M.A.; VIEGAS, K.A.S. et al. Aspectos morfológicos do ciclo testicular anual de codorna doméstica (Coturnix coturnix) da variedade italiana. Braz. J. Vet. Res. Anim. Sci, v.42, p.163-170, 2005.

SCHEIN, M.W.; DIAMOND, M.; CARTER, C.S. Sexual performance levels of male Japanese quail (Coturnix coturnix japonica). Anim. Behav., v.20, p.61-67, 1972. 